\title{
Kinematics of the Ultra-High-Velocity Gas in the Expanding Molecular Shell Adjacent to the W44 Supernova Remnant
}

\section{Masaya Yamada, Tomoharu Oka, Kunihiko Tanaka, Mariko Nomura, Shunya Takekawa, Yuhei Iwata, Sekito Tokuyama, Keisuke Tanabe, Shiho Tsujimoto and Maiko Furusawa}

Department of Physics, Faculty of Science and Technology, Keio University, 3-14-1 Hiyoshi, Kohoku-ku, Yokohama, Kanagawa 223-8522, Japan email: yamada@aysheaia.phys.keio.ac.jp

Keywords. ISM: clouds, ISM: molecules, (ISM:) supernova remnants, ISM: kinematics and dynamics,

\section{The "HVCCs" in the Galactic Center}

High-velocity compact cloud (HVCC) is a peculiar category of molecular clouds detected in the central molecular zone of our Galaxy (Oka et al. 1998, 2007, and 2012). They are characterized by compact appearances $(d<5 \mathrm{pc})$ and very large velocity widths $\left(\Delta V>50 \mathrm{~km} \mathrm{~s}^{-1}\right)$. Some of them show high $\mathrm{CO} J=3-2 / J=1-0$ intensity ratios $(\geqslant 1.5)$, indicating that they consist of dense and warm molecular gas. Dispite a number of efforts, we have not reached a comprehensive interpretation of HVCCs. Recently, we detected an extraordinaly broad velocity width feature, the 'Bullet', in the molecular cloud interacting with the W44 supernova remnant. The Bullet shares essential properties with HVCCs. Because of its proximity, a close inspection of the Bullet must contribute to the understanding of HVCCs.

\section{SNR W44 and the "Bullet"}

SNR W44 is a "mixed-morphology" supernova remnant (SNR) having a radio continuum shell filled with a center-peaked X-ray emission.It has a radio pulsar within the boundary of the shell. The distance to the SNR is considered to be $3 \mathrm{kpc}$ based on HI absorption studies. The W44 SNR is interacting with the adjacent giant molecular cloud (Seta et al. 1998, 2004). Shocked molecular gas is well traced by $25 \mathrm{OH} 1720 \mathrm{MHz}$ maser spots (Claussen et al. 1997), several highvelocity wings of $\mathrm{CO}$ emission and by a faint spatially-extended wing of $\mathrm{CO}$ and $\mathrm{HCO}^{+}$emissions. Such $\mathrm{OH}$ maser and wing emissions arise from a thin expanding shell of shocked molecular gas, of which the expansion velocity is $13.2 \mathrm{~km} \mathrm{~s}^{-1}$. The total kinetic energy is $E_{\text {kin }} \simeq 10^{50.1}$ erg (Sashida et al. 2013).

In the process of inspecting the kinematics of the W44 expanding shell, we noticed the Bullet at $(l, b)=\left(34.725^{\circ},-0.472^{\circ}\right)$ in the CO $J=3-2$ data. The full-width-zero-intensity velocity width exceeds $100 \mathrm{~km} \mathrm{~s}^{-1}$, while those of the wings are less than $30 \mathrm{~km} \mathrm{~s}^{-1}$. The position of the Bullet is $\sim 0.15^{\circ}$ ( $\sim 8 \mathrm{pc}$ in projected distance) apart from the $\mathrm{W} 44$ radio pulsar. The kinematics of the Bullet distinctly differs from that of the expanding molecular shell. Interestingly, a radio continuum blob (Figure 1b; Jones et al. 1993) and $\mathrm{H}_{2}$ ro-vibrational line nebulosity (Reach et al. 2005) overlap with the Bullet in the plane of the sky. Despite the high significance of the Bullet in the CO $J=3-2$ data, its nature and origin were left unexplained.

To unveil the origin of the Bullet, we performed detailed mappings in several millimeter and submillimeter lines using the Nobeyama Radio Observatory (NRO) $45 \mathrm{~m}$ radio telescope and the Atacama Submillimeter Telescope Experiment (ASTE) $10 \mathrm{~m}$ telescope. Our observation revealed that the Bullet clearly appears in the CO $J=1-0$, CO $J=3-2, \mathrm{CO} J=4-3$, and $\mathrm{HCO}^{+}$ 


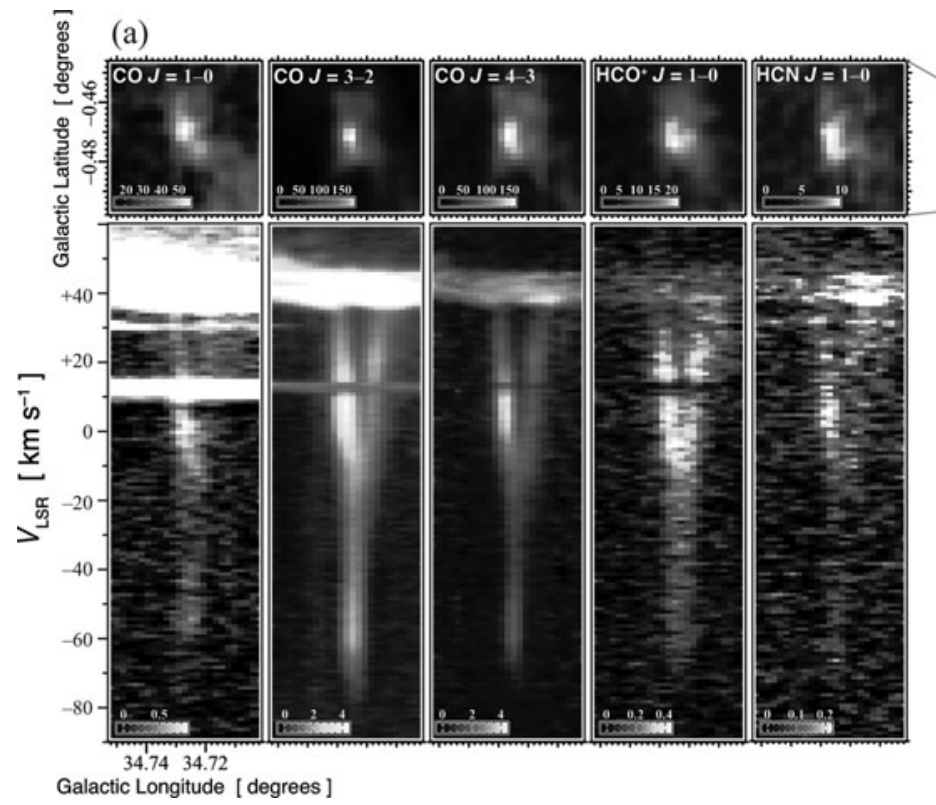

(b)
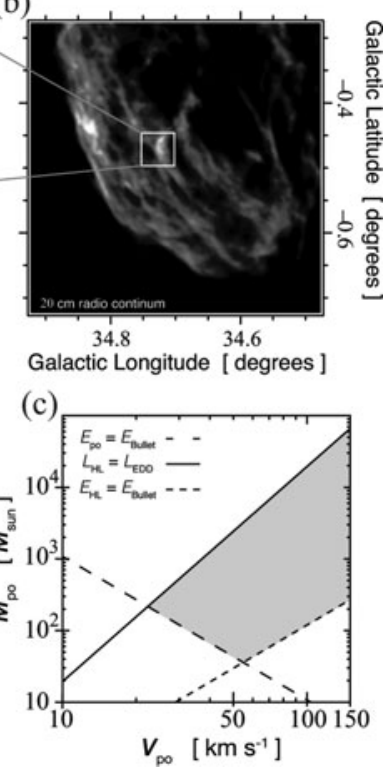

Figure 1. (a) (upper)Velocity-integrated maps of each emission, respectively. The velocity range for integration is $V_{\mathrm{LSR}}=-79-+34 \mathrm{~km} \mathrm{~s}^{-1}$. (lower)Longitude-velocity maps at the Galactic latitude of $-0.472^{\circ}$. (b)VLA radio continuum image at $\lambda=20 \mathrm{~cm}$. (c)Lines of $E_{\mathrm{po}}=E_{\mathrm{B} \text { ullet }}$, $E_{\mathrm{HL}}=E_{\mathrm{Bullet}}$ and $L_{\mathrm{HL}}=L_{\mathrm{Edd}}$ in the $M_{\mathrm{po}}-V_{\mathrm{po}}$ plane. Gray shaded area indicates the permitted range of parameters.

$J=1-0$ maps with a compact appearance $\left(0.5 \times 0.8 \mathrm{pc}^{2}\right)$ having an extremely broad velocity width $\left(\Delta V \simeq 120 \mathrm{~km} \mathrm{~s}^{-1}\right)$ and a very high CO $J=3-2 / J=1-0$ intensity ratio ( $\geqslant 3$ ) (Figure 1a).

\section{Our working hypothesis : Shooting Model}

Using the physical conditions derived for the Bullet and assuming the optically thin limit, we estimated the total mass of the Bullet to be $7.5 M_{\odot}$. If the Bullet arises from the W44 molecular cloud at $V_{\mathrm{LSR}} \simeq 40 \mathrm{~km} \mathrm{~s}^{-1}$, its kinetic energy amounts to $10^{48.0} \mathrm{erg}$. This kinetic energy is two orders of magnitude greater than the kinetic energy shared to the small solid angle of the Bullet, $10^{50} \mathrm{erg} \times(\Delta \Omega / 4 \pi)=10^{46.3} \mathrm{erg}$. Obviously, the energy injection by a SN explosion alone can not account for the Bullet. We consider a formation scenario for the Bullet along with the 'shooting' model:

(a) A massive compact object plunges into the W44 molecular cloud from the other side to this side.

(b) Ambient gas gravitates and converges in a wake behind the object.

(c) Gas in the wake accretes toward the object.

This process is known as the Bondi-Hoyle-Littleton (BHL) accretion (e.g., Edgar 2004). According to this scenario, the kinetic energy of the Bullet $\left(E_{\mathrm{B} u l l e t}\right)$ restricts the mass of the plunging object $\left(M_{\mathrm{po}}\right)$ and the shooting velocity $\left(V_{\mathrm{po}}\right)$. First, $E_{\mathrm{Bullet}}$ must be smaller than the initial kinetic energy of the plunging object. Second, $E_{\mathrm{B} \text { ullet }}$ must be smaller than the energy available from the BHL accretion. Another constraint comes from the condition that the luminosity $L_{\mathrm{HL}}$ can not exceed the Eddington limit $\left(L_{\mathrm{Edd}}\right)$. Figure 1c shows the range of $\left(M_{\mathrm{po}}, V_{\mathrm{po}}\right)$ permitted by $E_{\mathrm{po}} \geqslant E_{\mathrm{Bullet}}, E_{\mathrm{HL}} \geqslant E_{\mathrm{Bullet}}$, and $L_{\mathrm{HL}} \leqslant L_{\mathrm{Edd}}$, indicating that the plunging object must have a mass greater than $30 M_{\odot}$.

\section{References}

Oka, T., Hasegawa, T., Sato, F., Tsuboi, M., \& Miyazaki, A. 1998, ApJS, 118, 455 
Oka, T., Nagai, M., Kamegai, K., Tanaka, K., \& Kuboi, N. 2007, PASJ, 59, 15

Oka, T., Onodera, Y., Nagai, M., et al. 2012, ApJS, 201, 14

Seta, M., Hasegawa, T., Dame, T. M., et al. 1998, ApJ, 505, 286

Seta, M., Hasegawa, T., Sakamoto, S., et al. 2004, AJ, 127, 1098

Claussen, M. J., Frail, D. A., Goss, W. M., \& Gaume, R. A. 1997, ApJ, 489, 143

Sashida, T., Oka, T., Tanaka, K., et al. 2013, ApJ, 774, 10

Jones, L. R., Smith, A., \& Angelini, L. 1993, MNRAS, 265, 631

Reach, W. T., Rho, J., \& Jarrett, T. H. 2005, ApJ, 618, 297

Edgar, R. 2004, NewAR, 48, 843 